Published in final edited form as:

Nat Methods. 2016 October 31; 13(11): 897-898. doi:10.1038/nmeth.4030.

\title{
An acquisition and analysis pipeline for scanning angle interference microscopy
}

\author{
Catherine B Carbone ${ }^{1,2}$, Ronald D Vale ${ }^{1,2}$, and Nico Stuurman ${ }^{1,2}$ \\ Ronald D Vale: ron.vale@ucsf.edu \\ ${ }^{1}$ Department of Cellular and Molecular Pharmacology, University of California San Francisco, San \\ Francisco, California, USA \\ ${ }^{2}$ Howard Hughes Medical Institute, San Francisco, California, USA
}

\section{To the Editor}

We describe open-source software and hardware tools for calibrating, acquiring, and analyzing images for scanning angle interference microscopy (SAIM) analysis. These tools make it possible for a user with a total internal reflection fluorescence (TIRF) microscope equipped with a motorized illumination unit to generate reconstructed images with nanometer precision in the axial $(z)$ direction and diffraction-limited resolution in the lateral $(x, y)$ plane.

Surface-generated fluorescence interference contrast methods, including SAIM, can help researchers determine the axial position or topography of biological structures by reporting the average vertical position within a diffraction-limited $\operatorname{spot}^{1-3}$. These methods have a powerful ability to examine largely unexplored dynamics of thin structures like membranes, adhesion complexes, and the cytoskeleton with nanometer accuracy in the axial direction ${ }^{3}$. Though SAIM utilizes relatively simple optics that are available on commercial microscopes, difficulties with calibration and the lack of available analysis software have hampered its widespread adoption. Here, we describe a tool for automated angle calibration and a freely available, open-source software, which together create a straightforward pipeline for SAIM analysis.

\section{Code availability}

SAIM analysis and mManager acquisition software (Supplementary Software) are available under the Berkeley Software Distribution (BSD) license. Full documentation and examples are available at the project pages http://imagej.net/Saim and https://valelab.ucsf.edu/ $\sim$ kcarbone/SAIM. Development is hosted on GitHub at https://github.com/valelab/saimAnalysis and https://github.com/valelab/ saimCalibration.

Note: Any Supplementary Information and Source Data files are available in the online version of the paper (doi:10.1038/nmeth. 4030).

\section{AUTHOR CONTRIBUTIONS}

C.B.C., R.D.V., and N.S. designed the research; N.S. and C.B.C. developed the software and calibration device; C.B.C. collected data to test software; C.B.C. and N.S. drafted the article; C.B.C., R.D.V., and N.S. edited the article; and all authors read and approved the final article.

\section{COMPETING FINANCIAL INTERESTS}

The authors declare competing financial interests: details are available in the online version of the paper (doi:10.1038/nmeth.4040). 
For interference contrast microscopy, the sample is placed on a transparent spacer on top of a mirror (silicon wafer with silicon oxide spacer; Fig. 1a and Supplementary Methods). Incident excitation light interferes with its own reflection (Fig. 1b), resulting in an intensity field that varies with axial distance in a theoretically predictable manner (Supplementary Note 1). Upon varying the angle of excitation, different intensity profiles can be obtained. By acquiring images at multiple angles and fitting the intensities pixel by pixel to the theoretical prediction, one can determine the average height of each spatial element with nanometer accuracy.

Our SAIM pipeline begins with an automated calibration procedure (Fig. 1c, left; and Supplementary Note 2). We designed a simple apparatus, which is controlled with a plugin for the open-source $\mu$ Manager software ${ }^{4}$ that defines the relation between motor position and angle of illumination. This device sits above the objective at the position of the sample. It consists of a transparent acrylic front plate that fluoresces upon illumination by a laser beam and two vertically spaced linear charge-coupled devices (CCDs) that localize the beam as it passes through the front plate (Supplementary Protocol). Compared with manual procedures, automated angle measurement provides a faster, more precise calibration. Automated calibration is especially advantageous for multicolor measurements, as it reduces the time required to calibrate each laser by about ten-fold. Furthermore, automated calibration is highly reproducible, minimizing errors in calibration, which can readily translate into height measurement errors of greater than $10 \mathrm{~nm}$ (Supplementary Fig. 1).

After calibration, the sample is imaged at varying angles of illumination by controlling a motorized illuminator with the $\mu$ Manager plugin (Fig. 1c, middle) ${ }^{4}$. Next, these images are analyzed to generate a topographical map (Fig. 1c, right). Our analysis software is a Fiji ${ }^{5}$ plugin that (i) plots the predicted excitation intensity at a given distance from the surface of the oxide layer as a function of angle, (ii) fits the theoretical model to an experimentally determined intensity profile at a given region of interest (ROI), and (iii) carries out fits for all pixels in an image (Supplementary Note 3 ). The calibration and acquisition plugin is independent from the analysis plugin; however, they can be used within the same package because they both run in ImageJ.

Samples for SAIM are prepared on silicon substrates with an oxide spacer. To measure absolute height of a sample, it is critical to know the precise thickness of the oxide spacer. We measured the oxide thickness of SAIM substrates using ellipsometry (Supplementary Fig. 2b). We also estimated oxide spacer thickness using SAIM (Supplementary Fig. 2b,c). We recommend performing this measurement on the day of an experiment to minimize variability that is caused by changes in the microscope and/or calibrations (Supplementary Fig. 2d and Supplementary Note 4). Once calibrated, this technique can be used to measure the height of a lipid bilayer with a standard deviation around $1 \mathrm{~nm}$ at approximately $10^{6}$ photons per pixel (Fig. 1d,e and Supplementary Fig. 2e).

To test accuracy and limitations of SAIM and our analysis pipeline, we measured nanometer-scale height changes in samples of biomolecules with known sizes (also see Supplementary Note 5 and Supplementary Fig. 3). First, we measured the deformation of a microtubule (25-nm diameter) as it crossed on top of an axoneme (a rigid structure of 
microtubules with a diameter of about $200 \mathrm{~nm}$ ). The average microtubule height change at an axoneme crossing was measured by our SAIM technique to be $227 \pm 44 \mathrm{~nm}(n=6$, one replicate; Fig. 1f, modeled in Fig. 1g). Next, we measured the deformation of a lipid membrane at a membrane interface consisting of a giant unilamellar vesicle interacting with a supported lipid bilayer and separated by protein spacers of two different heights $(5 \mathrm{~nm}$ for FRB-rapamycin-FKBP and $18 \mathrm{~nm}$ for a $3 \mathrm{X}$-mCherry; Fig. 1h). The height difference caused these proteins to segregate from one another into distinct domains (Fig. 1i) (ref. 6). The membrane height change at a $3 \mathrm{X}$-mCherry cluster relative to an adjacent FRBrapamycin-FKBP domain was $13.7 \pm 6.0 \mathrm{~nm}$ ( $n=157$ clusters from five giant unilamellar vesicle (GUVs) imaged during two separate experiments; Fig. 1i,j), as measured by our SAIM pipeline. This result is consistent with the expected height difference (Fig. 1h) and similar to values obtained by reflection interference contrast microscopy for an analogous membrane deformation experiment ${ }^{6}$.

These open-source tools should greatly facilitate the implementation of SAIM for studying the axial organization of fluorescently tagged molecules at nanometer scales.

\section{Supplementary Material}

Refer to Web version on PubMed Central for supplementary material.

\section{Acknowledgments}

We would like to thank G. Bhabha and S. Niekamp for help with electron microscopy, axoneme and microtubule experiments and J. Sung and S. Vo (director of the Leia3d Advance Lithography Center in Palo Alto, California) for ellipsometry measurements. We thank T. Goddard and E. Meng for help generating topography models of SAIM data using Chimera software. We are grateful to V. Weaver and M. Rubashkin for initial help implementing SAIM and to M. Paszek and W. Shin for useful discussions about the SAIM software and calibration device. We thank G. Bhabha and A. Jain for comments on the manuscript. The authors acknowledge funding from the National Institutes of Health (R01EB007187, R.D.V.) and the Howard Hughes Medical Institute.

\section{References}

1. Lambacher A, Fromherz P. Appl Phys A. 1996; 63:207-216.

2. Ajo-Franklin CM, Ganesan PV, Boxer SG. Biophys J. 2005; 89:2759-2769. [PubMed: 16085775]

3. DuFort C, Paszek M. Methods Cell Biol. 2014; 123:235-252. [PubMed: 24974031]

4. Edelstein, A.; Amodaj, N.; Hoover, K.; Vale, R.; Stuurman, N. Current Protocols in Molecular Biology. Ausubel, FM., editor. John Wiley \& Sons, Inc; 2010. Unit 14.20

5. Schindelin J, et al. Nat Methods. 2012; 9:676-682. [PubMed: 22743772]

6. Schmid EM, et al. Nat Phys. 2016; 12:704-711. 

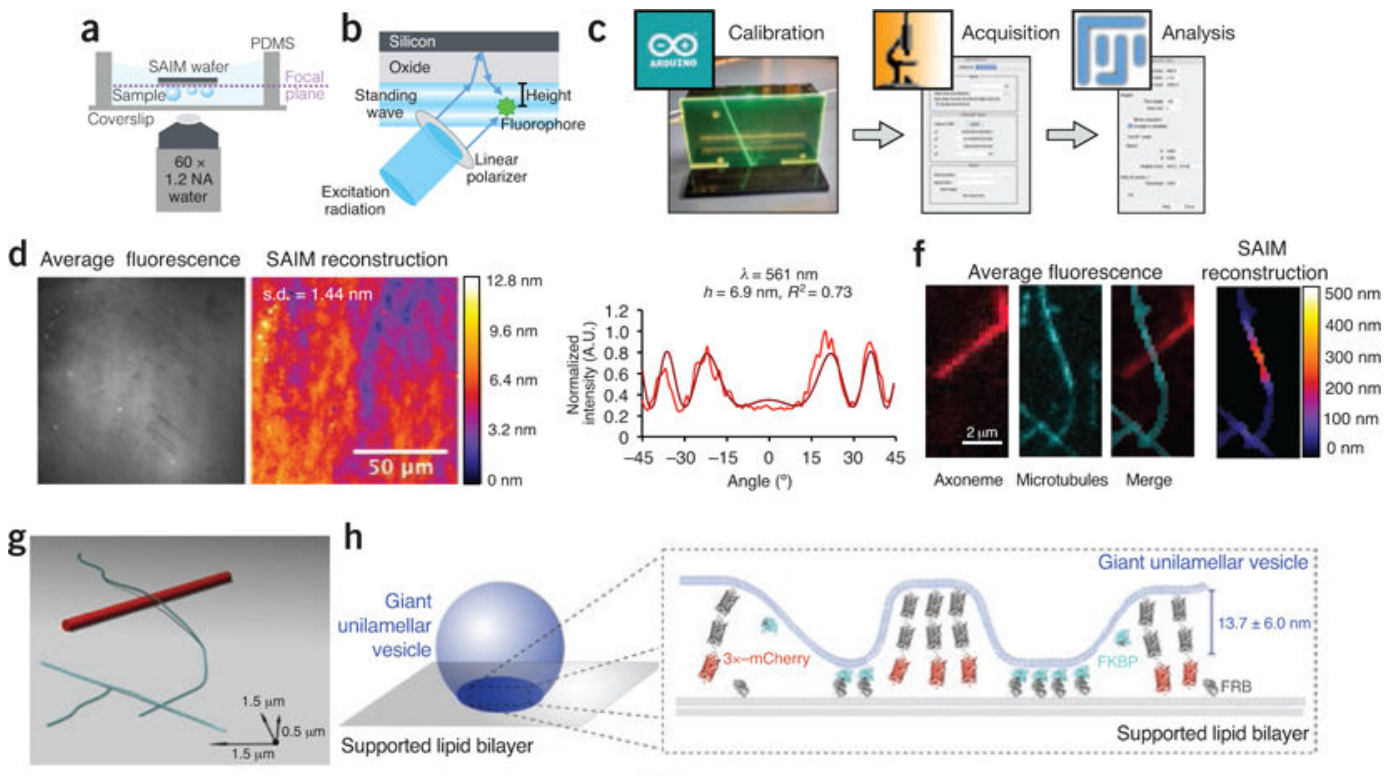

h

i

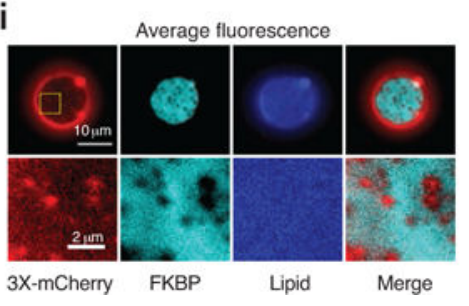

Giant unilamellar
vesicle

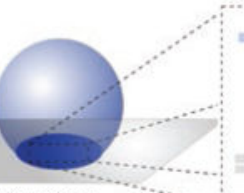

Axoneme Microtubules Merge

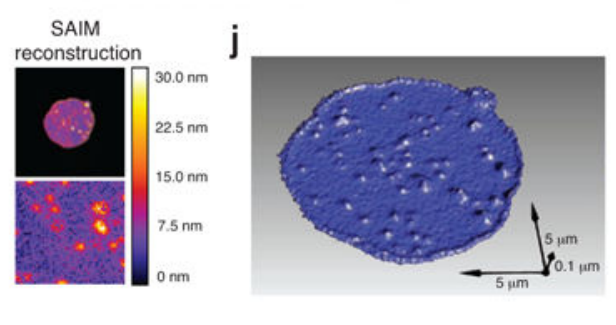

Figure 1.

Implementation of SAIM pipeline to measure sample topography. (a) Schematic representation of sample setup for SAIM. NA, numerical aperture; PDMS, polydimethylsiloxane. (b) Schematic of SAIM theory. (c) Schematic of SAIM tools. (d) SAIM height of a supported lipid bilayer labeled with DiI. Left, average fluorescence intensity across all angles sampled. Right, SAIM reconstruction showing a height s.d. of $1.44 \mathrm{~nm}$ across a $10,000-\mu \mathrm{m}^{2}$ field. Relation between color and height is shown in bar at the right of SAIM heightmap. (e) Representative single-pixel fit for supported lipid bilayer measurements. Raw data shown in light red; fit shown in dark red. A.U., arbitrary units. $\lambda$, wavelength; $h$, pixel height obtained from fit; $R^{2}$, coefficient of determination. (f) SAIM height of a microtubule crossing an axoneme. Left three images, average fluorescence intensity for a Cy5-labeled axoneme (red) and Alexa-647-labeled microtubule (cyan). Right, SAIM reconstruction of microtubule height showing a representative deflection at the microtubule's intersection with the axoneme and intersection with other microtubules. (g) 3D model of data shown in $\mathbf{f}$. Microtubules are shown as cylinders with a radius of $12.5 \mathrm{~nm}$ along spline fits through SAIM data (cyan). The axoneme is modeled as a straight cylinder with a radius of $100 \mathrm{~nm}$ (red). Axes represent $x^{-}, y$-, and $z$-scales. (h) Schematic of membrane interface showing relative sizes of proteins used to form partitioned membrane regions based on published Protein Data Bank structures 2h5q and 3fap. (i) SAIM height of a membrane interface between a giant unilamellar vesicle (GUV) and a supported lipid bilayer (SLB). Left, average fluorescence intensity for a GUV-tethered triple-length mCherry (red), GUV-tethered Alexa-647-labeled FKBP binding SLB-tethered FRB (cyan), 
Atto390-labeled GUV membrane (blue) and the merged mCherry and FKBP fluorescence images. Right, SAIM reconstruction of GUV membrane derived from Atto390-labeled lipid fluorescence showing representative deflection at mCherry clusters. (j) 3D model of data shown in $\mathbf{i}$. $Z$-scale is exaggerated to clearly depict membrane deformations. 